\title{
Scale-Dependent Nonaffine Elasticity of Semiflexible Polymer Networks
}

\author{
M. Atakhorrami, ${ }^{1, *}$ G. H. Koenderink, ${ }^{1,2}$ J. F. Palierne, ${ }^{3}$ F. C. MacKintosh, ${ }^{1, \dagger}$ and C. F. Schmidt ${ }^{1,4, \$}$ \\ ${ }^{1}$ Department of Physics and Astronomy, VU University, De Boelelaan 1081, 1081 HV Amsterdam, Netherlands \\ ${ }^{2}$ FOM Institute AMOLF, Science Park 104, XG Amsterdam, Netherlands \\ ${ }^{3}$ École Normale Supérieure de Lyon, Laboratoire de Physique, 15 Parvis René Descartes, 69342 Lyon Cedex, France \\ ${ }^{4}$ Third Institute of Physics, Georg August University, Friedrich-Hund-Platz 1, 37077 Göttingen, Germany
}

(Received 30 October 2012; published 24 February 2014)

\begin{abstract}
The cytoskeleton of eukaryotic cells provides mechanical support and governs intracellular transport. These functions rely on the complex mechanical properties of networks of semiflexible protein filaments. We study the impact of local network deformations on the scale-dependent mobility of probe particles in entangled networks of actin filaments using high-bandwidth microrheology. We find that micron-sized particles in these networks experience two opposing noncontinuum elastic effects: entropic depletion reduces the effective network rigidity, while local nonaffine deformations of the network substantially enhance the rigidity at low frequencies, eventually leading to a size-independent response and strong violation of the generalized Stokes formula. We show that a simple model of lateral bending of filaments embedded in a viscoelastic background leads to an intermediate scaling regime for the apparent elastic modulus $G^{\prime}(\omega) \sim \omega^{9 / 16}$, closely matching the experiments. These results demonstrate that nonaffine bending deformations can be dominant for the mobility of objects of the size of vesicles and organelles in the cell.
\end{abstract}

DOI: 10.1103/PhysRevLett.112.088101

PACS numbers: 87.10.Pq, 87.16.Ka, 87.16.Ln, 87.85.jc

Living cells are highly structured composites containing a multicomponent polymer network, the cytoskeleton, consisting of the filamentous proteins F-actin, microtubules, and intermediate filaments [1-3]. Characteristic length scales range from monomer sizes of $\sim 50 \AA$ through network mesh sizes of $\sim 100 \mathrm{~nm}$ to filament persistence lengths of $\mu \mathrm{m}$ to $\mathrm{mm}$. The mechanical response of the cytoskeleton therefore depends on the time and length scales on which it is probed. Vesicles and organelles, for instance, which move within the cytoskeleton, are tens of nanometers to micrometers in size, and therefore experience local response that may differ substantially from continuum mechanics. Elastic moduli inside cells have been estimated by video microrheology, analyzing fluctuations of probe particles [4]. The structural complexity of cells and intrinsic nonequilibrium fluctuations, however, can confound such measurements [4,5]. Microrheological studies have also been performed on reconstituted cytoskeletal networks, in particular F-actin [6-15]. Even simple F-actin networks show a discrepancy between local and bulk response [6,7,11,15-17], the physical origin of which has remained obscure. The large persistence length of cytoskeletal polymers implies that filament bending will be part of the local response. Such nonaffine deformations have been treated theoretically and in simulations [18-23]. Direct evidence of nonaffine effects in cytoskeletal networks, however, has been limited [24], and the impact of nonaffinity on local mechanical properties is not known.

Here we combine bulk rheology with microrheology measurements using optically trapped beads of various sizes to study the scale-dependent mechanics of cytoskeletal F-actin networks with high bandwidth. We use dual optical tweezers to measure material response with twoparticle microrheology (2p MR) [16,25], which infers bulk properties from particle-pair correlations and which is insensitive to the beads' local surroundings or bead size $[16,25]$. We compare this, in the same experiment, with one-particle microrheology (1p MR) [12,14], which is sensitive to local response.

We find evidence for two local noncontinuum elastic effects. The particles introduce local pockets of reduced polymer concentration by entropic depletion, leading to a reduction of the local modulus. Surprisingly, however, the local stiffness at intermediate and low frequencies is enhanced. Such an effect has been suggested to exist on theoretical grounds [26] in the pure elastic limit, as a result of filament bending, but this has never been verified. We introduce a model for frequency-dependent nonaffine effects due to bending of filaments, which leads to an apparent scaling regime $G^{\prime}(\omega) \sim \omega^{9 / 16}$, closely matching the experiments. At even lower frequencies, the response converges toward a particle-size independent local stiffness for small particles.

Samples were prepared by polymerizing rabbit skeletal muscle actin in F-buffer ( $2 \mathrm{~m} M$ HEPES, $p \mathrm{H}$ 7.5, $50 \mathrm{mM}$ $\mathrm{KCl}, 2 \mathrm{mM} \mathrm{MgCl}$, and $1 \mathrm{~m} M \mathrm{Na}_{2}$ ATP [8], chemicals from Sigma/Aldrich, USA). Actin was polymerized for $\sim 1 \mathrm{~h}$ in glass and double-stick-tape chambers with silica probe beads (Kisker Biotech, Germany, radii $R=0.26,0.51,1.28$, and $2.5 \mu \mathrm{m}$, polydispersity $5 \%$ ). The particles were larger than the average network mesh 


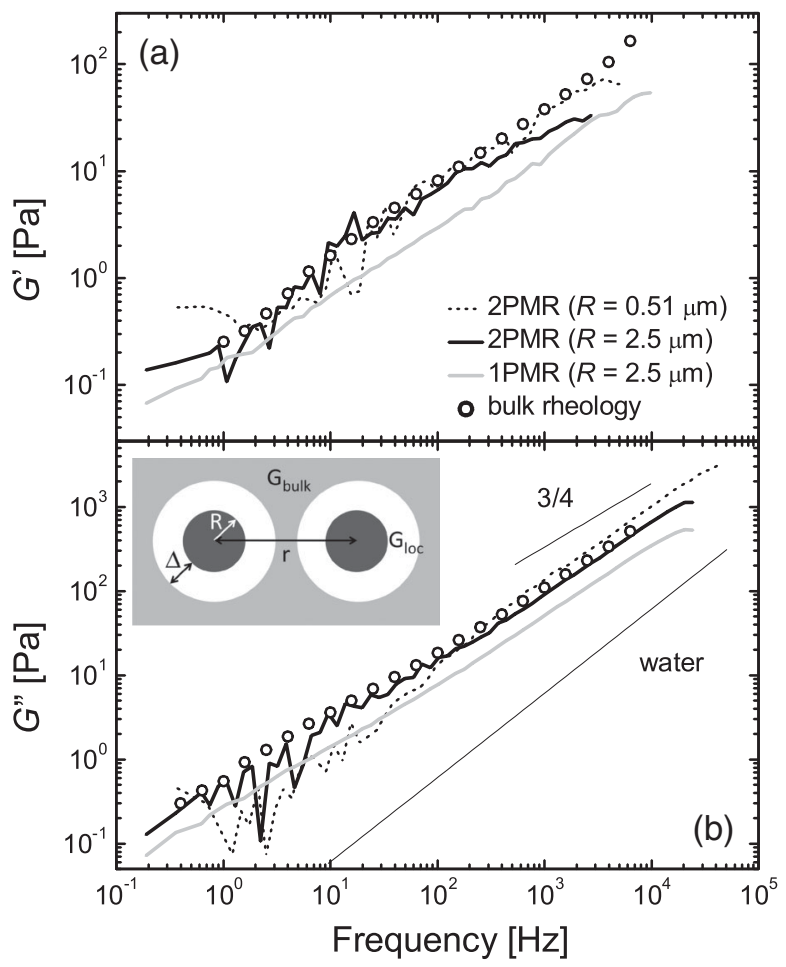

FIG. 1 Local shear elastic moduli of entangled solutions of $1 \mathrm{mg} / \mathrm{ml} \mathrm{F}$-actin are smaller than macroscopic moduli: (a) storage moduli $G^{\prime}(\omega)$, (b) loss moduli $G^{\prime \prime}(\omega)$. Shear moduli from 2p MR (solid line: particle radius $R=2.5 \mu \mathrm{m}$; dashed line: $R=0.51 \mu \mathrm{m}$, particle distances $10-15 \mu \mathrm{m}$ ) agree with bulk rheology (open circles); 1p MR measures smaller moduli (gray: $R=2.5 \mu \mathrm{m}$ ). Power-law slope of $3 / 4$ indicated, solvent viscosity contribution, $G_{\text {water }}^{\prime \prime}(\omega)$, also indicated. Inset: schematic of probe particles (radius $R$ ) at distance $r$, with depletion shell of thickness $\Delta$.

size, $\xi \approx 0.3 / \sqrt{c_{A}}$ (in $\mu \mathrm{m}$, with concentration in $\mathrm{mg} / \mathrm{ml}$ ) [27]. Actin concentrations $c_{A}$ were in the range $0.5-2 \mathrm{mg} / \mathrm{ml}$. Bulk rheology was performed in a custom-built piezorheometer $[28,29]$ by oscillating one glass plate sinusoidally $(0.1 \mathrm{~Hz}<f<10 \mathrm{kHz})$ normal to its surface with an amplitude of $1 \mathrm{~nm}$. Stress transmitted to a second parallel plate was measured by a piezoelectric element. Squeezing flow leads to a shear strain amplitude of $\sim 10^{-6}$, ensuring linear response. Microrheology was performed with a custom-built dual optical tweezers setup [30]. Pairs of beads, at least 10 bead diameters away from any surface, were weakly trapped using near-IR lasers $(1064$ and $830 \mathrm{~nm})$ with trap stiffness below $3 \times 10^{-6} \mathrm{~N} / \mathrm{m}$. Position fluctuations were detected by quadrant photodiodes at a sampling rate of $195 \mathrm{kHz}$ and converted to storage and loss shear moduli using the fluctuation-dissipation theorem and the generalized Stokes relation [12]. 1p MR moduli represent averages over $x$ and $y$ displacements of particles, while 2p MR moduli were derived from the correlated motions of two particles parallel to the line connecting their centers. Fluid inertia was negligible for the separation distances $r$ used here $(r=10-15 \mu \mathrm{m})[31,32]$. We typically took data of 10 bead pairs for each distance. Each experiment was reproduced at least three times with new samples. Thus $1 \mathrm{p}$ MR data are averaged over $>40$ data sets, $2 \mathrm{p}$ MR data over $>10$ data sets for each distance. As seen before [12], the distribution of response functions in different locations and samples was relatively narrow (data not shown).

Storage and loss shear moduli $G^{\prime}(\omega)$ and $G^{\prime \prime}(\omega)$ from 2p MR were independent of particle size and agreed well with macroscopic moduli [Figs. 1(a) and 1(b)], confirming that $2 \mathrm{p}$ MR reliably measures bulk properties. In contrast, shear moduli obtained from $1 p$ MR were significantly smaller than the macroscopic moduli [Figs. 1(a) and 1(b)], even for the largest particles $(R=2.5 \mu \mathrm{m})$, which are 17 -fold bigger in diameter than the mesh size $(\sim 300 \mathrm{~nm})$ but still smaller than average filament length $(\sim 20 \mu \mathrm{m})$.

To understand the origin of the difference between local and bulk response, we used particles of different sizes. With reduced particle radii of 2.5 to $0.26 \mu \mathrm{m}, G^{\prime \prime}(\omega)$ from 1p MR decreased further in amplitude (Fig. 2), consistent with earlier studies of actin networks [6,7,11,15-17]. At frequencies $>10 \mathrm{kHz}$, both the local and macroscopic loss moduli scaled with frequency as $G^{\prime \prime}(\omega) \propto \omega^{3 / 4}$ [Fig. 1(b)]. In this regime, the viscoelastic response is dominated by the relaxation of thermal bending fluctuations of individual filament segments between entanglement points $[8,33]$. Here, moduli are expected to be proportional to polymer concentration. Thus, lower local moduli suggest a reduced polymer concentration near the probe particles. The likely explanation for this is entropic depletion: particles restrict conformations of polymers. Such an effect has been observed for flexible synthetic polymers

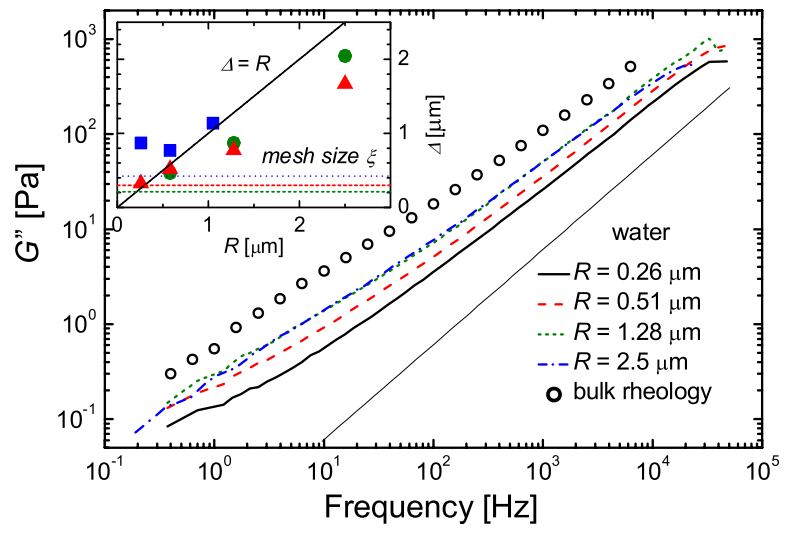

FIG. 2 (color online). Loss moduli from $1 \mathrm{p}$ MR reveal actin depletion near beads. $G^{\prime \prime}(\omega)$ for $1 \mathrm{mg} / \mathrm{ml}$ F-actin. 1p MR: particle radii $R=0.26,0.51,1.28,2.5 \mu \mathrm{m}$ (lines), bulk rheology (open circles). Solvent viscosity is indicated. Inset: $\Delta$ calculated from microrheology data $>10 \mathrm{kHz}$, plotted versus particle radius, actin concentrations $c_{A}=0.5$ (squares), 1 (triangles), and $2 \mathrm{mg} / \mathrm{ml}$ (circles). Horizontal lines mark network mesh sizes: dotted 0.5 , dashed 1 , dash-dotted $2 \mathrm{mg} / \mathrm{ml}$; solid line is particle radius $R$. 
[34] and DNA [35]. The thickness of the depletion zone $\Delta$ can be estimated from local and macroscopic moduli at high frequencies [17,35]. Our 1p MR data can be mapped onto the $2 \mathrm{p}$ MR data using a simplified depletion model: each particle is assumed to be surrounded by a sharply defined, completely polymer-depleted shell of solvent of thickness $\Delta$ [25] [Fig. 1(b), inset]. The thickness $\Delta$ was calculated [36] from the ratio $G^{\prime \prime}(\omega)^{2 \mathrm{p} \mathrm{MR}} / G^{\prime \prime}(\omega)^{1 \mathrm{pMR}}$ at frequencies $\omega>10 \mathrm{kHz}$ using Eq. (3) from Ref. [35], which was derived in [37], in which the medium surrounding the bead is modeled as a viscous shell of thickness $\Delta$ that is surrounded by a stiffer viscoelastic continuum. We find that $\Delta$ grows with particle size (Fig. 2, inset), consistent with previous reports [17]. Around spherical particles in a solution of semiflexible polymers, $\Delta$ is expected to be of order the particle size $[38,39]$. Near a flat wall, $\Delta$ is predicted to be of order the polymer length or persistence length, whichever is shorter [40]. This depletion range exceeds that seen with entangled flexible polymers, where $\Delta \approx$ mesh size $[34,35]$. Local microrheology measurements for cytoskeletal polymer networks are therefore always affected by depletion, unless polymers are attracted by the particles [10].

The local storage modulus $G^{\prime}(\omega)$ measured with 1p MR exhibits a stronger and more complex particle-size dependence than the loss modulus. At high frequencies, $G^{\prime}(\omega)$ from $1 \mathrm{p}$ MR is smaller than the macroscopic modulus, similar to $G^{\prime \prime}(\omega)$ [Fig. 3(a)]. At low frequencies, however, the smallest particles ( 0.26 and $0.51 \mu \mathrm{m}$ radius), experience a local elastic modulus apparently exceeding the macroscopic shear modulus. Moreover, the local response exhibits a weaker frequency dependence. These surprising observations suggest a second noncontinuum effect in the interaction with the surrounding network. We hypothesize that this effect is due to nonaffine local network deformations resulting from the transverse bending of filaments as the particle moves. Bending penetrates into the network to a depth determined by a balance between filament bending and elastic deformation of the network $[19,26]$. Moving particles push against these stiff filaments embedded in a matrix, considered here for simplicity as an elastic continuum. For a displacement $u$, the bending energy is $E_{\text {bending }} \sim n \kappa u^{2} / \lambda^{3}$, where $\lambda$ is the length over which a filament of bending modulus $\kappa$ bends. The number $n$ of bent filaments increases with the particle size as $n \sim R / \xi$. To obtain this estimate, we assume that only filaments oriented nearly perpendicular to the axis of motion are bent because, with only steric interactions between particles and filaments, tangential forces on the bead are negligible. The particle cross section scales as $R^{2}$, and network mesh density seen along an axis as $1 / \xi^{2}$. Each filament extends over of order $R / \xi$ meshes. Hence, the number of displaced filaments as the particle moves along one axis scales as $R / \xi$. Assuming $\lambda \gg R$, the elastic energy stored in the distorted surrounding elastic continuum is $E_{\text {elastic }} \sim G u^{2} \lambda$, where $G$ is the network shear modulus [41]. Minimizing the sum of these energies results in a characteristic deflection length [42],
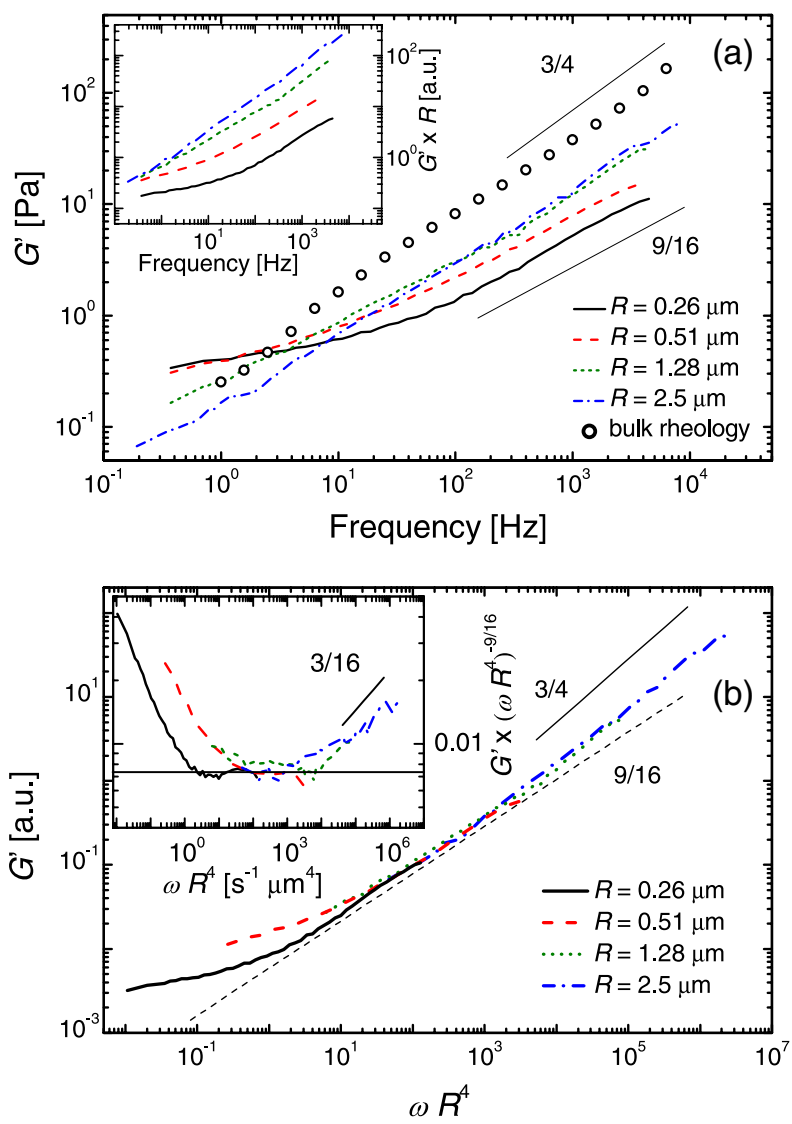

FIG. 3 (color online). Storage moduli $G^{\prime}(\omega)$ from 1p MR reveal local nonaffine bending of actin filaments. (a) $G^{\prime}(\omega)$ for $1 \mathrm{mg} / \mathrm{ml}$ F-actin. 1p MR: particle radii $R=0.26,0.51,1.28,2.5 \mu \mathrm{m}$ (lines), bulk rheology (open circles). $3 / 4$ slope indicates expected high frequency scaling, 9/16 slope expected from our model. Inset: Convergence of $G^{\prime}(\omega) \times R$ at low frequencies. (b) Apparent elastic moduli determined from $1 \mathrm{p}$ MR with different bead sizes, shifted to account for depletion, plotted against frequency normalized by $2 \pi R^{4}$, showing agreement with $\omega^{9 / 16}$ scaling (dashed line). Shift factors were consistent with those observed between 1p MR $G^{\prime \prime}$ curves at high frequencies due to depletion, corrected for the frequency-axis rescaling. Inset: same data with $G^{\prime}(\omega)$ multiplied by $\left(\omega R^{4}\right)^{-9 / 16}, \omega^{9 / 16}$ scaling at intermediate and $\omega^{3 / 4}$ scaling at high frequencies.

$$
\lambda_{0}=\sqrt[4]{\frac{\kappa R}{G \xi}}
$$

A similar length scale $\lambda_{b}=\sqrt[4]{\kappa / G}$ was found for bending [43,44] and buckling [45] of microtubules in the cytoskeleton. Our probe particle experiences a total restoring force $f \sim-G u \lambda_{0}$. In stiffer media, $\lambda_{0}$ will decrease and will eventually become smaller than particle radius $R$. In that case, the matrix dominates the fibers, and the restoring force will become that of a particle in an elastic continuum: $f \sim-G u R$. Thus, a transition is expected, when $\lambda_{0} \sim R$, between particle-size independent response, dominated by nonaffine filament bending, and (particle-size dependent) "normal response." It is qualitatively expected that $G^{\prime}(\omega)$ is more strongly affected by 
local response than $G^{\prime \prime}(\omega)$. At the lower measured frequencies, the macroscopic $G^{\prime}$ is still smaller than the macroscopic $G^{\prime \prime}$ since a plateau has not been reached. Because of local bending of the primarily elastic filaments, there will be an enhanced value of $G^{\prime}$. This must occur together with an enhancement of $G^{\prime \prime}$, but the relative effect on $G^{\prime}$ will be greater. Since the network shear modulus increases with frequency, a transition to a local bending-dominated regime should occur below a characteristic frequency $\omega_{0}$. To estimate this frequency, we assume that the network modulus follows a power law $G=A c_{A} \omega^{3 / 4}$, as is typically found for actin networks [33], with amplitude $A$. Using Eq. (1), the crossover frequency below which the filament bending effects should become apparent, is then of order

$$
\omega_{0} \sim\left(\frac{\kappa}{c_{A} \xi R^{3}}\right)^{4 / 3}
$$

As long as $\lambda_{0}$ remains larger than $R$, the probe bead acts as if it were covered with rigid "bristles" due to the stiff filaments that spread the force out. In other words, for frequencies $\omega<\omega_{0}$, the bead will behave as if its size were $\lambda_{0}$. The $1 \mathrm{p}$ MR analysis, which assumes particle radius $R$, will then report an increased local (apparent) modulus,

$$
G_{\text {local }} \sim \frac{G \lambda}{R} \sim \frac{\left(c_{A} \omega\right)^{9 / 16}}{R^{3 / 4}} .
$$

Our model thus predicts a local elastic modulus with a low frequency dependence of $\omega^{9 / 16}$, which should cross over to bulk response with $\omega^{3 / 4}$ scaling.

Our data are well explained by this model. The elastic modulus [Fig. 3(a)] is consistent with a $\omega^{9 / 16}$ scaling for the two smallest probes $(R=0.26$ and $0.51 \mu \mathrm{m})$ over about two decades. For the intermediate-size particles $(R=1.28 \mu \mathrm{m})$ we find $\omega^{9 / 16}$ scaling over the whole frequency range. For the largest particles $(R=2.5 \mu \mathrm{m}), G^{\prime}(\omega) \sim \omega^{3 / 4}$, like the macroscopic modulus. The effect of nonaffine deformations can be confirmed by collapsing all curves for the different particle sizes onto a master curve by simultaneously rescaling $\omega$ by $R^{4}$, which is proportional to $\omega_{0}$ in Eq. (2), and shifting the curves vertically to account for depletion [Fig. 3 (b)]. The resulting curve scales as $\omega^{9 / 16}$ over a large frequency range and approaches $\omega^{3 / 4}$ at high frequencies, which is emphasized by plotting $\left(\omega R^{4}\right)^{-9 / 16} G^{\prime}(\omega)$ against $\omega R^{4}$ [Fig. 3(b), inset]. The resulting curve is flat at intermediate frequencies and crosses over to $3 / 16$ scaling at high frequencies, consistent with $G^{\prime} \sim \omega^{3 / 4}$.

For the two smaller beads, the slope decreases further at the lowest frequencies. Such a phenomenon can be understood, even if no elastic plateau is yet reached. This may indicate a distinct low frequency regime governed by a nonaffine length scale $\lambda$ that saturates to a large length scale. If this length is intrinsic and independent of the particle size, then Eq. (3) would result in an apparent stiffness $G_{\text {local }}$ that varies inversely with particle size, corresponding to particle fluctuations that are independent of size. Interestingly, this is consistent with what we see in the inset to Fig. 3(a), where $G$ multiplied with $R$ is plotted. Such a convergence requires that the $9 / 16$ regime gives way at low frequencies to a weaker dependence on frequency, corresponding to a stiffening of the response, relative to the intermediate $9 / 16$ regime, at lower frequencies. As the length $\lambda_{0}$ also grows toward lower frequencies, this stiffening may occur when the transverse filament bending extends over a range larger than the bead-radiusdependent depletion zone. It is worth noting that the observed behavior at the lowest frequencies is particularly relevant to the standard video range covered by many passive microrheology methods.

Our results demonstrate that the local response of a semiflexible polymer network can strongly violate the generalized Stokes formula for a bulk continuum medium. Such local noncontinuum effects are particularly relevant in biopolymer networks, where intrinsic length scales such as filament persistence length are on the micrometer scale. At low frequencies $(<10-100 \mathrm{~Hz})$ one might also expect a difference between 1p MR and bulk rheology due to possible contributions of compressibility $[12,14,25]$. The resulting additional compliance from compressibility on small scales is expected to be visible primarily in $1 \mathrm{p}$ MR rather than $2 \mathrm{p}$ MR. The effects we observe at low frequencies, however, have the opposite trend, i.e., showing less compliance in $1 \mathrm{p} \mathrm{MR}$, which excludes a dominance of compressibility. Our simple model accounts quantitatively for the frequency-dependent reduction in the mobility of the probes due to local bending deformations. Interestingly, when translating the anomalous response to an apparent elastic modulus, this model predicts scaling as $\omega^{9 / 16}$, very close to the $\omega^{1 / 2}$ scaling that has frequently been reported $[13,46]$, but has thus far remained unexplained. Our observations are at odds with prior suggestions that $1 \mathrm{p}$ MR can accurately probe bulk rheology at low frequencies [7]. An implication of our work is that it is not appropriate to locally treat a semiflexible polymer network as a continuum, even when probe particles are substantially larger than the mesh size. This is crucial to understand before one extends quantitative microrheology to the complex cytoplasm of cells. Organelles and transport vesicles, for instance, feel a response that is likely to be dominated by the local nonaffine bending deformations of the rather stiff cytoskeletal polymers.

We thank A. J. Levine, D. A. Head, F. Gittes, D. C. Morse, M. Pasquali, K. M. Addas, J. Liu, D. A. Weitz, and P. A. Janmey for helpful discussions, J. van Mameren, J. I. Kwiecińska, and M. Buchanan for software development, and K. C. Vermeulen for actin preparation. This work was supported by the Dutch Foundation for Fundamental Research on Matter (FOM). Additional support for C. F. S. was provided by the German Science Foundation (DFG) Center for the Molecular Physiology of the Brain (CMPB) and by the DFG Collective Research Center SFB 937, 
Project No. A2. This work was supported in part by the National Science Foundation under Grant No. PHYS1066293 and the hospitality of the Aspen Center for Physics.

*Present address: University College London, Maple House 1A, 149 Tottenham Court Road, London W1T 7NF, United Kingdom.

${ }^{\dagger}$ Corresponding author.

fcm@nat.vu.nl

${ }^{\ddagger}$ Corresponding author.

cfs@physik3.gwdg.de

[1] E. M. De La Cruz, A. Mandinova, M. O. Steinmetz, D. Stoffler, U. Aebi, and T. D. Pollard, J. Mol. Biol. 295, 517 (2000).

[2] H. Isambert, P. Venier, A. C. Maggs, A. Fattoum, R. Kassab, D. Pantaloni, and M. F. Carlier, J. Biol. Chem. 270, 11437 (1995).

[3] F. Gittes, B. Mickey, J. Nettleton, and J. Howard, J. Cell Biol. 120, 923 (1993).

[4] A. W. C. Lau, B. D. Hoffmann, A. Davies, J. C. Crocker, and T. C. Lubensky, Phys. Rev. Lett. 91, 198101 (2003).

[5] D. Mizuno, C. Tardin, C. F. Schmidt, and F. C. MacKintosh, Science 315, 370 (2007).

[6] B. S. Chae and E. M. Furst, Langmuir 21, 3084 (2005).

[7] M. L. Gardel, M. T. Valentine, J. C. Crocker, A. R. Bausch, and D. A. Weitz, Phys. Rev. Lett. 91, 158302 (2003).

[8] G. H. Koenderink, M. Atakhorrami, F. C. MacKintosh, and C. F. Schmidt, Phys. Rev. Lett. 96, 138307 (2006).

[9] H. Lee, J. M. Ferrer, F. Nakamura, M. J. Lang, and R. D. Kamm, Acta Biomater. 6, 1207 (2010).

[10] J. L. McGrath, J. H. Hartwig, and S. C. Kuo, Biophys. J. 79, 3258 (2000).

[11] F. G. Schmidt, B. Hinner, and E. Sackmann, Phys. Rev. E 61, 5646 (2000).

[12] B. Schnurr, F. Gittes, F. C. MacKintosh, and C. F. Schmidt, Macromolecules 30, 7781 (1997).

[13] F. Ziemann, J. Radler, and E. Sackmann, Biophys. J. 66, 2210 (1994).

[14] F. Gittes, B. Schnurr, P. D. Olmsted, F. C. MacKintosh, and C. F. Schmidt, Phys. Rev. Lett. 79, 3286 (1997).

[15] J. Liu, M. L. Gardel, K. Kroy, E. Frey, B. D. Hoffman, J. C. Crocker, A. R. Bausch, and D. A. Weitz, Phys. Rev. Lett. 96, 118104 (2006).

[16] J. C. Crocker, M. T. Valentine, E. R. Weeks, T. Gisler, P. D. Kaplan, A. G. Yodh, and D. A. Weitz, Phys. Rev. Lett. 85, 888 (2000).

[17] J. Y. Huh and E. M. Furst, Phys. Rev. E 74, 031802 (2006).

[18] D. A. Head, A. J. Levine, and F. C. MacKintosh, Phys. Rev. Lett. 91, 108102 (2003).

[19] D. A. Head, F. C. MacKintosh, and A. J. Levine, Phys. Rev. E 68, 025101 (2003).

[20] C. Heussinger and E. Frey, Phys. Rev. Lett. 97, 105501 (2006).

[21] C. Heussinger and E. Frey, Phys. Rev. E 75, 011917 (2007).

[22] C. P. Broedersz, X. M. Mao, T. C. Lubensky, and F. C. MacKintosh, Nat. Phys. 7, 983 (2011).

[23] P. R. Onck, T. Koeman, T. van Dillen, and E. van der Giessen, Phys. Rev. Lett. 95, 178102 (2005).

[24] J. Liu, G. H. Koenderink, K. E. Kasza, F. C. MacKintosh, and D. A. Weitz, Phys. Rev. Lett. 98, 198304 (2007).

[25] A. J. Levine and T. C. Lubensky, Phys. Rev. Lett. 85, 1774 (2000).
[26] A. C. Maggs, Phys. Rev. E 57, 2091 (1998).

[27] C. F. Schmidt, M. Barmann, G. Isenberg, and E. Sackmann, Macromolecules 22, 3638 (1989).

[28] M. Cagnon and G. Durand, Phys. Rev. Lett. 45, 1418 (1980).

[29] M. Buchanan, M. Atakhorrami, J. F. Palierne, and C. F. Schmidt, Macromolecules 38, 8840 (2005).

[30] M. Atakhorrami, K. M. Addas, and C. F. Schmidt, Rev. Sci. Instrum. 79, 043103 (2008).

[31] M. Atakhorrami, G. H. Koenderink, C. F. Schmidt, and F. C. MacKintosh, Phys. Rev. Lett. 95, 208302 (2005).

[32] M. Atakhorrami, D. Mizuno, G. H. Koenderink, T. B. Liverpool, F. C. MacKintosh, and C. F. Schmidt, Phys. Rev. E 77, 061508 (2008).

[33] F. Gittes and F. C. MacKintosh, Phys. Rev. E 58, R1241 (1998).

[34] L. Starrs and P. Bartlett, Faraday Discuss. 123, 323 (2003).

[35] D. T. Chen, E. R. Weeks, J. C. Crocker, M. F. Islam, R. Verma, J. Gruber, A. J. Levine, T. C. Lubensky, and A. G. Yodh, Phys. Rev. Lett. 90, 108301 (2003).

[36] This equation $G_{2 p} / G_{1 p}=\left(4 \beta^{6} \kappa^{\prime 2}-9 \beta^{5} \kappa \kappa^{\prime}+10 \beta^{3} \kappa \kappa^{\prime}-\right.$ $\left.9 \beta \kappa^{\prime 2}-15 \beta \kappa^{\prime}+2 \kappa \kappa^{\prime \prime}\right) /\left[2\left(\kappa^{\prime \prime}-2 \beta^{5} \kappa^{\prime}\right)\right]$ relates the ratio between the shear moduli measured with one $\left(G_{1 p}\right)$ and two particles $\left(G_{2 p}\right)$, using the depletion shell thickness $(\Delta)$ and the ratio between macroscopic shear modulus $\left(G_{\text {macro }}\right)$ and that of the shell material $\left(G_{\mathrm{loc}}\right)$. It assumes incompressibility. Here $\beta=R /(R+\Delta), \kappa=G_{\text {macro }} / G_{\text {loc }}, \kappa^{\prime}=\kappa-1 ; \kappa^{\prime \prime}=3+2 \kappa$. It is derived by calculating the displacement field in the whole composite of shell and surrounding medium when a force is exerted on one particle, matching displacements and stresses at the interface between shell and surrounding medium. $G_{2 p}$ is derived similarly calculating the displacement of a second particle when a force is exerted on the first particle [37].

[37] A. J. Levine and T. C. Lubensky, Phys. Rev. E 65, 011501 (2001).

[38] D. C. Morse, Macromolecules 31, 7044 (1998).

[39] Y. L. Chen and K. S. Schweizer, J. Phys. Chem. B 108, 6687 (2004).

[40] D. C. Morse and G. H. Fredrickson, Phys. Rev. Lett. 73, 3235 (1994).

[41] We estimate $E_{\text {elastic }}$ as follows. With force $f$, the far-field displacement and strain at distance $r$ scale as $f / G r$ and $f / G r^{2}$, while $E_{\text {elastic }} \propto G \int\left(f /\left(G r^{2}\right)\right)^{2} r^{2} d r$. This integral is dominated by the lower limit, which is $\lambda$. By equating the displacement at $r=\lambda$ with the bead displacement, we obtain $E_{\text {elastic }} \propto G u^{2} \lambda$ and $f \propto G \lambda u$. Assuming the deflection is primarily in the plane perpendicular to bead motion, the prefactor in the force will not be the $6 \pi$ of Stokes drag, but dependence on $\lambda$ is as above.

[42] L. D. Landau, E. M. Lifshitz, A. M. Kosevich, and L. P. Pitaevskii, Theory of Elasticity (Pergamon Press, Oxford, 1986).

[43] C. P. Brangwynne, F. C. MacKintosh, and D. A. Weitz, Proc. Natl. Acad. Sci. U.S.A. 104, 16128 (2007).

[44] C. P. Brangwynne, G. H. Koenderink, F. C. MacKintosh, and D. A. Weitz, Phys. Rev. Lett. 100, 118104 (2008).

[45] C. P. Brangwynne, F. C. MacKintosh, S. Kumar, N. A. Geisse, J. Talbot, L. Mahadevan, K. K. Parker, D. E. Ingber, and D. A. Weitz, J. Cell Biol. 173, 733 (2006).

[46] J. Uhde, W. Feneberg, N. Ter-Oganessian, E. Sackmann, and A. Boulbitch, Phys. Rev. Lett. 94, 198102 (2005). 